\title{
Weighted Lipschitz estimates for commutators on weighted Morrey spaces
}

\section{Yan Lin*, Zongguang Liu and Wenli Cong}

\section{"Correspondence:}

linyan@cumtb.edu.cn

School of Sciences, China University

of Mining and Technology, Beijing,

100083, P.R. China

\begin{abstract}
In this paper, the authors establish the boundedness of commutators generated by weighted Lipschitz functions and strongly singular Calderón-Zygmund operators or generalized Calderón-Zygmund operators on weighted Morrey spaces.
\end{abstract}

MSC: 42B20; 42B25; 42B35

Keywords: strongly singular Calderón-Zygmund operator; weighted Lipschitz function; generalized Calderón-Zygmund operator; weighted Morrey space

\section{Introduction}

The study of generalized Calderón-Zygmund operators and strongly singular nonconvolution operators originated in the classical Calderón-Zygmund operator. The theory of the Calderón-Zygmund operator was one of the important achievements of classical analysis in the last century, and it has many important applications in Fourier analysis, complex analysis, operator theory and so on.

The introduction of the strongly singular Calderón-Zygmund operator is motivated by a class of multiplier operators whose symbol is given by $e^{i|\xi|^{a}} /|\xi|^{\beta}$ away from the origin, $0<a<1, \beta>0$. Fefferman and Stein [1] enlarged the multiplier operators onto a class of convolution operators. Coifman [2] also considered a related class of operators for $n=1$. The strongly singular non-convolution operator, whose properties are similar to those of the classical Calderón-Zygmund operator, but the kernel is more singular near the diagonal than that of the standard one, was introduced by Alvarez and Milman in [3]. Furthermore, following a suggestion of Stein, the authors in [3] showed that the pseudodifferential operator with symbol in the Hörmander class $S_{\alpha, \delta}^{-\beta}$, where $0<\delta \leq \alpha<1$ and $n(1-\alpha) / 2 \leq \beta<n / 2$, is included in the strongly singular Calderón-Zygmund operator. Thus, the strongly singular Calderón-Zygmund operator correlates closely with both the theory of Calderón-Zygmund singular integrals in harmonic analysis and the theory of pseudo-differential operators in partial differential equations.

Suppose that $T$ is a strongly singular Calderón-Zygmund operator or a generalized Calderón-Zygmund operator, whose strict definitions will be given later, and $b$ is a locally integrable function on $\mathbf{R}^{n}$. The commutator $[b, T]$ generated by $b$ and $T$ is defined as follows:

$$
[b, T](f)(x)=b(x) T f(x)-T(b f)(x) .
$$

(c) 2015 Lin et al. This article is distributed under the terms of the Creative Commons Attribution 4.0 International License (http://creativecommons.org/licenses/by/4.0/), which permits unrestricted use, distribution, and reproduction in any medium, provided you give appropriate credit to the original author(s) and the source, provide a link to the Creative Commons license, and indicate if changes were made. 
In 1986, Alvarez and Milman [3, 4] discussed the boundedness of the strongly singular Calderón-Zygmund operator on Lebesgue spaces. In 2006, in [5], the behavior of Toeplitz operators related to strongly singular Calderón-Zygmund operators and Lipschitz functions was discussed on Lebesgue spaces. Furthermore, in 2007, Lin and Lu [6] proved the boundedness of the commutators of strongly singular Calderón-Zygmund operators on Hardy type spaces. In 2008, the authors in [7] obtained two kinds of endpoint estimates for strongly singular Calderón-Zygmund operators. And the pointwise estimate for the sharp maximal function of commutators generated by strongly singular Calderón-Zygmund operators and $B M O$ functions was also established.

In 2007, the authors in [8] obtained the boundedness of generalized Calderón-Zygmund operators on weighted Lebesgue spaces and weighted Hardy spaces. In 2011, the pointwise estimates for the sharp maximal functions of commutators generated by generalized Calderón-Zygmund operators and $B M O$ functions or Lipschitz functions were established in [9].

The classical Morrey spaces which were introduced by Morrey in [10] came from [10, 11] to study the local behavior of solutions to second order elliptic partial differential equations. For the properties and applications of classical Morrey spaces, one can refer to [10, 12]. In 1987, Chiarenza and Frasca [13] proved the boundedness of the Hardy-Littlewood maximal operator, the fractional integral operator and the Calderón-Zygmund singular integral operator on Morrey spaces. In 2010, Fu and Lu [14] established the boundedness of weighted Hardy operators and their commutators on Morrey spaces. Lin $[9,15]$ discussed the commutators of strongly singular Calderón-Zygmund operators and generalized Calderón-Zygmund operators on Morrey spaces, respectively.

In 2009, Komori and Shirai [16] defined the weighted Morrey spaces and studied the boundedness of the Hardy-Littlewood maximal operator, the fractional integral operator and the classical Calderón-Zygmund singular integral operator on these weighted spaces. In 2012, Wang [17] researched the behavior of commutators generated by classical Calderón-Zygmund operators and weighted Lipschitz functions or weighted BMO functions on weighted Morrey spaces. In 2013, the authors in [18] proved the boundedness of some sublinear operators and their commutators on weighted Morrey spaces. In 2014, Lin and Sun [19] established the boundedness of commutators generated by strongly singular Calderón-Zygmund operators and weighted $B M O$ functions on weighted Morrey spaces. In 2015, the authors in [20] studied the properties of commutators generated by generalized Calderón-Zygmund operators and weighted $B M O$ functions on weighted Morrey spaces.

Inspired by the above results, in this paper we are interested in the boundedness of the commutators generated by weighted Lipschitz functions and strongly singular CalderónZygmund operators or generalized Calderón-Zygmund operators on weighted Morrey spaces.

Before stating our main results, let us first recall some necessary definitions and notations.

Definition 1.1 Let $\mathcal{S}$ be the space of all Schwartz functions on $\mathbf{R}^{n}$ and $\mathcal{S}^{\prime}$ be its dual space, the class of all tempered distributions on $\mathbf{R}^{n}$. Let $T: \mathcal{S} \rightarrow \mathcal{S}^{\prime}$ be a bounded linear operator. $T$ is called a strongly singular Calderón-Zygmund operator if the following three conditions are satisfied. 
(1) $T$ can be extended into a continuous operator from $L^{2}\left(\mathbf{R}^{n}\right)$ into itself.

(2) There exists a function $K(x, y)$ continuous away from the diagonal $\{(x, y): x=y\}$ such that

$$
|K(x, y)-K(x, z)|+|K(y, x)-K(z, x)| \leq C \frac{|y-z|^{\delta}}{|x-z|^{n+\frac{\delta}{\alpha}}},
$$

if $2|y-z|^{\alpha} \leq|x-z|$ for some $0<\delta \leq 1$ and $0<\alpha<1$. And

$$
\langle T f, g\rangle=\iint K(x, y) f(y) g(x) d y d x
$$

for $f, g \in \mathcal{S}$ with disjoint supports.

(3) For some $n(1-\alpha) / 2 \leq \beta<n / 2$, both $T$ and its conjugate operator $T^{*}$ can be extended into continuous operators from $L^{q}$ to $L^{2}$, where $1 / q=1 / 2+\beta / n$.

Definition 1.2 Suppose that $T: \mathcal{S} \rightarrow \mathcal{S}^{\prime}$ is a linear operator with kernel $K(\cdot, \cdot)$ defined initially by

$$
T(f)(x)=\int_{\mathbf{R}^{n}} K(x, y) f(y) d y, \quad f \in C_{c}^{\infty}\left(\mathbf{R}^{n}\right), x \notin \operatorname{supp} f .
$$

The operator $T$ is called a generalized Calderón-Zygmund operator provided the following three conditions are satisfied.

(1) $T$ can be extended into a continuous operator on $L^{2}\left(\mathbf{R}^{n}\right)$.

(2) $K$ is smooth away from the diagonal $\{(x, y): x=y\}$ with

$$
\int_{|x-y|>2|z-y|}(|K(x, y)-K(x, z)|+|K(y, x)-K(z, x)|) d x \leq C,
$$

where $C>0$ is a constant independent of $y$ and $z$.

(3) There is a sequence of positive constant numbers $\left\{C_{j}\right\}$ such that for each $j \in \mathbf{N}$,

$$
\left(\int_{2^{j}|z-y| \leq|x-y|<2^{j+1}|z-y|}|K(x, y)-K(x, z)|^{q} d x\right)^{1 / q} \leq C_{j}\left(2^{j}|z-y|\right)^{-n / q^{\prime}}
$$

and

$$
\left(\int_{2^{j}|y-z| \leq|y-x|<2^{j+1}|y-z|}|K(y, x)-K(z, x)|^{q} d x\right)^{1 / q} \leq C_{j}\left(2^{j}|z-y|\right)^{-n / q^{\prime}},
$$

where $\left(q, q^{\prime}\right)$ is a fixed pair of positive numbers with $1 / q+1 / q^{\prime}=1$ and $1<q^{\prime}<2$.

If we compare the generalized Calderón-Zygmund operator with the classical CalderónZygmund operator, whose kernel $K(x, y)$ enjoys the conditions

$$
|K(x, y)| \leq C|x-y|^{-n}
$$

and

$$
|K(x, y)-K(x, z)|+|K(y, x)-K(z, x)| \leq C|x-y|^{-n}\left(\frac{|z-y|}{|x-y|}\right)^{\delta},
$$


where $|x-y|>2|z-y|$ for some $\delta>0$, we can find out that the classical Calderón-Zygmund operator is a generalized Calderón-Zygmund operator defined as in Definition 1.2 with $C_{j}=2^{-j \delta}, j \in \mathbf{N}$, and any $1<q<\infty$.

Definition 1.3 ([21]) A non-negative measurable function $\omega$ is said to be in the Muckenhoupt class $A_{p}$ with $1<p<\infty$ if for every cube $Q$ in $\mathbf{R}^{n}$, there exists a positive constant $C$ independent of $Q$ such that

$$
\left(\frac{1}{|Q|} \int_{Q} \omega(x) d x\right)\left(\frac{1}{|Q|} \int_{Q} \omega(x)^{1-p^{\prime}} d x\right)^{p-1} \leq C
$$

where $Q$ denotes a cube in $\mathbf{R}^{n}$ with the side parallel to the coordinate axes and $1 / p+1 / p^{\prime}=1$. When $p=1$, a non-negative measurable function $\omega$ is said to belong to $A_{1}$ if there exists a constant $C>0$ such that for any cube $Q$,

$$
\frac{1}{|Q|} \int_{Q} \omega(y) d y \leq C \omega(x), \quad \text { a.e. } x \in Q \text {. }
$$

It is well known that if $\omega \in A_{p}$ with $1<p<\infty$, then $\omega \in A_{r}$ for all $r>p$ and $\omega \in A_{q}$ for some $1<q<p$.

Definition 1.4 ([16]) Let $1 \leq p<\infty, 0<k<1$ and $\omega$ be a weighted function. Then the weighted Morrey space $L^{p, k}(\omega)$ is defined by

$$
L^{p, k}(\omega)=\left\{f \in L_{\mathrm{loc}}^{p}(\omega):\|f\|_{L^{p, k}(\omega)}<\infty\right\}
$$

where

$$
\|f\|_{L^{p, k}(\omega)}=\sup _{Q}\left(\frac{1}{\omega(Q)^{k}} \int_{Q}|f(x)|^{p} \omega(x) d x\right)^{1 / p},
$$

and the supremum is taken over all cubes $Q$ in $\mathbf{R}^{n}$.

Definition 1.5 ([16]) Let $1 \leq p<\infty, 0<k<1$ and $u$, $v$ be two weighted functions. Then the weighted Morrey space $L^{p, k}(u, v)$ for two weights is defined by

$$
L^{p, k}(u, v)=\left\{f \in L_{\mathrm{loc}}^{p}(u, v):\|f\|_{L^{p, k}(u, v)}<\infty\right\},
$$

where

$$
\|f\|_{L^{p, k}(u, v)}=\sup _{Q}\left(\frac{1}{v(Q)^{k}} \int_{Q}|f(x)|^{p} u(x) d x\right)^{1 / p}
$$

and the supremum is taken over all cubes $Q$ in $\mathbf{R}^{n}$.

Definition 1.6 Let $1 \leq p<\infty, 0<\beta_{0}<1$ and $\omega$ be a weighted function. A locally integrable function $b$ is said to be in the weighted Lipschitz space $\operatorname{Lip}_{\beta_{0}}^{p}(\omega)$ if

$$
\|b\|_{\operatorname{Lip}_{\beta_{0}}^{p}(\omega)}=\sup _{Q} \frac{1}{\omega(Q)^{\beta_{0} / n}}\left(\frac{1}{\omega(Q)} \int_{Q}\left|b(x)-b_{Q}\right|^{p} \omega(x)^{1-p} d x\right)^{1 / p}<\infty
$$


where $b_{Q}=\frac{1}{|Q|} \int_{Q} b(y) d y$ and the supremum is taken over all cubes $Q \subset \mathbf{R}^{n}$. Moreover, we denote simply by $\operatorname{Lip}_{\beta_{0}}(\omega)$ when $p=1$.

Definition 1.7 The Hardy-Littlewood maximal operator $M$ is defined by

$$
M(f)(x)=\sup _{Q \ni x} \frac{1}{|Q|} \int_{Q}|f(y)| d y .
$$

We set $M_{s}(f)=M\left(|f|^{s}\right)^{1 / s}$, where $0<s<\infty$.

The sharp maximal operator $M^{\sharp}$ is defined by

$$
M^{\sharp}(f)(x)=\sup _{Q \ni x} \frac{1}{|Q|} \int_{Q}\left|f(y)-f_{Q}\right| d y \sim \sup _{Q \ni x} \inf _{a \in \mathbf{C}} \frac{1}{|Q|} \int_{Q}|f(y)-a| d y .
$$

We define the $t$-sharp maximal operator $M_{t}^{\sharp}(f)=M^{\sharp}\left(|f|^{t}\right)^{1 / t}$, where $0<t<1$.

Definition 1.8 ([22]) A weighted function $\omega$ belongs to the reverse Hölder class $R H_{r}$ if there exist two constants $r>1$ and $C>0$ such that the following reverse Hölder inequality

$$
\left(\frac{1}{|Q|} \int_{Q} \omega(x)^{r} d x\right)^{\frac{1}{r}} \leq C\left(\frac{1}{|Q|} \int_{Q} \omega(x) d x\right)
$$

holds for every cube $Q$ in $\mathbf{R}^{n}$. Denote by $r_{\omega}$ the critical index of $\omega$ for the reverse Hölder condition. That is, $r_{\omega}=\sup \left\{r>1: \omega \in R H_{r}\right\}$.

Definition 1.9 For $0<\beta_{0}<n, 1 \leq r<\infty$, the fractional maximal operator $M_{\beta_{0}, r}$ is defined by

$$
M_{\beta_{0}, r}(f)(x)=\sup _{Q \ni x}\left(\frac{1}{|Q|^{1-\beta_{0} r / n}} \int_{Q}|f(y)|^{r} d y\right)^{\frac{1}{r}}
$$

Definition 1.10 For $0<\beta_{0}<n, 1 \leq r<\infty$ and a weight $\omega$, the weighted fractional maximal operator $M_{\beta_{0}, r, \omega}$ is defined by

$$
M_{\beta_{0}, r, \omega}(f)(x)=\sup _{Q \ni x}\left(\frac{1}{\omega(Q)^{1-\beta_{0} r / n}} \int_{Q}|f(y)|^{r} \omega(y) d y\right)^{\frac{1}{r}},
$$

where the above supremum is taken over all cubes $Q$ containing $x$.

\section{Main results}

In what follows, we will give the main results in this paper.

Theorem 2.1 Let $T$ be a strongly singular Calderón-Zygmund operator, $\alpha, \beta, \delta$ be given as in Definition 1.1, and $\frac{n(1-\alpha)}{2}<\beta<\frac{n}{2}(n \geq 2)$. Suppose $0<\beta_{0}<1, \frac{n(1-\alpha)+2 \beta}{2 \beta}<p<\frac{n}{\beta_{0}}, \frac{1}{q}=\frac{1}{p}-\frac{\beta_{0}}{n}$, $0<k<\frac{p}{q}, \omega \in A_{1}$ and $r_{\omega}>\frac{(n(1-\alpha)+2 \beta)(p-1)}{2 \beta p-n(1-\alpha)-2 \beta}$. If $b \in \operatorname{Lip}_{\beta_{0}}(\omega)$, then $[b, T]$ is bounded from $L^{p, k}(\omega)$ to $L^{q, k q / p}\left(\omega^{1-q}, \omega\right)$.

Theorem 2.2 Let $T$ be a generalized Calderón-Zygmund operator and $q^{\prime}$ be given as in Definition 1.2. Suppose $0<\beta_{0}<1, q^{\prime}<p<n / \beta_{0}, 1 / s=1 / p-\beta_{0} / n, 0<k<p / s,\left\{j C_{j}\right\} \in l^{1}$, 
$\omega^{s / p} \in A_{1}$ and $r_{\omega}>\max \left\{\frac{(p-1) q^{\prime}}{p-q^{\prime}}, \frac{1-k}{p / s-k}\right\}$. If $b \in \operatorname{Lip}_{\beta_{0}}(\omega)$, then $[b, T]$ is bounded from $L^{p, k}(\omega)$ to $L^{s, k s / p}\left(\omega^{1-s}, \omega\right)$.

Noticing that the classical Calderón-Zygmund operator is a generalized CalderónZygmund operator defined as in Definition 1.2 with $C_{j}=2^{-j \delta}(j \in \mathbf{N})$ and any $1<q<\infty$, we can obtain the following result as a corollary.

Corollary 2.1 Let $T$ be a classical Calderón-Zygmund operator, $0<\beta_{0}<1,1<p<n / \beta_{0}$, $1 / s=1 / p-\beta_{0} / n, 0<k<p / s, \omega^{s / p} \in A_{1}$ and $r_{\omega}>\frac{1-k}{p / s-k}$. If $b \in \operatorname{Lip}_{\beta_{0}}(\omega)$, then $[b, T]$ is bounded from $L^{p, k}(\omega)$ to $L^{s, k s / p}\left(\omega^{1-s}, \omega\right)$.

Remark 2.1 As matter of fact, the result of Corollary 2.1 was obtained in [17] with the special case $\delta=1$. Thus Theorem 2.2 can be regarded as a generalization of the corresponding result in [17]. And from this point of view, the range of the index in Theorem 2.2 is reasonable.

\section{Preliminaries}

In order to obtain our main results, first we introduce some requisite lemmas.

Lemma $3.1([3,4])$ If $T$ is a strongly singular Calderón-Zygmund operator, then $T$ is the type of weak $(1,1)$ and can be defined to be a continuous operator from $L^{\infty}$ to BMO.

It follows from Definition 1.1, Lemma 3.1 and the interpolation theory that the strongly singular Calderón-Zygmund operator $T$ is bounded on $L^{p}$ for $1<p<\infty$, and $T$ is bounded from $L^{u}$ to $L^{v}, \frac{n(1-\alpha)+2 \beta}{2 \beta} \leq u<\infty$ and $0<\frac{u}{v} \leq \alpha$. In particular, if we restrict $\frac{n(1-\alpha)}{2}<\beta<\frac{n}{2}$ in (3) of Definition 1.1, then $T$ is bounded from $L^{u}$ to $L^{v}$, where $\frac{n(1-\alpha)+2 \beta}{2 \beta}<u<\infty$ and $0<\frac{u}{v}<\alpha$.

Lemma 3.2 ([8]) Let $T$ be a generalized Calderón-Zygmund operator and the sequence $\left\{C_{j}\right\} \in l^{1}$, then $T$ is bounded on $L^{p}\left(\mathbf{R}^{n}\right)$ and the type of weak $(1,1)$, where $1<p<\infty$.

Lemma 3.3 ([17]) Let $0<t<1,1<p<\infty$ and $0<k<1$. If $u, v \in A_{\infty}$, then we have

$$
\left\|M_{t}(f)\right\|_{L^{p, k}(u, v)} \leq C\left\|M_{t}^{\sharp}(f)\right\|_{L^{p, k}(u, v)}
$$

for all functions $f$ such that the left-hand side is finite. In particular, when $u=v=\omega$ and $\omega \in A_{\infty}$, we have

$$
\left\|M_{t}(f)\right\|_{L^{p, k}(\omega)} \leq C\left\|M_{t}^{\sharp}(f)\right\|_{L^{p, k}(\omega)}
$$

for all functions $f$ such that the left-hand side is finite.

Lemma 3.4 ([23]) Let $\mu \in A_{1}$, then there are constants $C_{1}, C_{2}$ and $0<\delta<1$ depending only on $A_{1}$-constant of $\mu$ such that for any measurable subset $E$ of a ball $B$,

$$
C_{1} \frac{|E|}{|B|} \leq \frac{\mu(E)}{\mu(B)} \leq C_{2}\left(\frac{|E|}{|B|}\right)^{\delta} .
$$


Lemma 3.5 ([19]) Let $T$ be a strongly singular Calderón-Zygmund operator, and $\alpha, \beta, \delta$ be given as in Definition 1.1. If $\frac{n(1-\alpha)+2 \beta}{2 \beta}<p<\infty, 0<k<1$ and $\omega \in A_{2 \beta p /[n(1-\alpha)+2 \beta]}$, then $T$ is bounded on $L^{p, k}(\omega)$.

Lemma 3.6 ([20]) Let $T$ be a generalized Calderón-Zygmund operator, $q^{\prime}$ be the same as in Definition 1.2, and the sequence $\left\{C_{j}\right\} \in l^{1}$. If $q^{\prime}<p<\infty, 0<k<1$ and $\omega \in A_{p / q^{\prime}}$, then $T$ is bounded on $L^{p, k}(\omega)$.

Lemma 3.7 If $\varepsilon>0$, then $\ln x \leq \frac{1}{\varepsilon} x^{\varepsilon}$ for all $x \geq 1$.

The above result comes from the monotone property of the function $\varphi(x)=\ln x-\frac{1}{\varepsilon} x^{\varepsilon}$.

Lemma 3.8 ([17]) Let $0<\beta_{0}<n, 1<p<n / \beta_{0}, 1 / s=1 / p-\beta_{0} / n$ and $\omega^{s / p} \in A_{1}$. Then if $0<k<p / s$ and $r_{\omega}>\frac{1-k}{p / s-k}$, we have

$$
\left\|M_{\beta_{0}, 1}(f)\right\|_{L^{s, k s / p}\left(\omega^{s / p}, \omega\right)} \leq C\|f\|_{L^{p, k}(\omega)} .
$$

Lemma 3.9 ([17]) Let $0<\beta_{0}<n, 1<p<n / \beta_{0}, 1 / s=1 / p-\beta_{0} / n, 0<k<p / s$ and $\omega \in A_{\infty}$. Then, for every $1<r<p$, we have

$$
\left\|M_{\beta, r, \omega}(f)\right\|_{L^{s, k s / p}(\omega)} \leq C\|f\|_{L^{p, k}(\omega)} .
$$

Lemma $3.10([24,25])$ Let $0<\beta_{0}<1$ and $\omega \in A_{1}$. Then, for any $1 \leq p<\infty$, there exists an absolute constant $C>0$ such that $\|b\|_{\operatorname{Lip}_{\beta_{0}}^{p}(\omega)} \leq C\|b\|_{\operatorname{Lip}_{\beta_{0}}(\omega)}$.

Lemma 3.11 Let $0<\beta_{0}<1, \omega \in A_{1}$ and $f$ be a function in $\operatorname{Lip}_{\beta_{0}}(\omega)$. Suppose $1 \leq p<\infty$, $x \in \mathbf{R}^{n}$, and $r_{1}, r_{2}>0$. Then

$$
\begin{aligned}
& \left(\frac{1}{\left|B\left(x, r_{1}\right)\right|} \int_{B\left(x, r_{1}\right)}\left|f(y)-f_{B\left(x, r_{2}\right)}\right|^{p} \omega(y)^{1-p} d y\right)^{\frac{1}{p}} \\
& \quad \leq C\|f\|_{\operatorname{Lip}_{\beta_{0}}(\omega)} \omega(x)\left(1+\left|\ln \frac{r_{2}}{r_{1}}\right|\right)\left(\frac{\omega\left(B\left(x, r_{1}\right)\right)}{\left|B\left(x, r_{1}\right)\right|}\right)^{-\frac{1}{p^{\prime}}} \max _{i=1,2} \omega\left(B\left(x, r_{i}\right)\right)^{\beta_{0} / n} .
\end{aligned}
$$

Proof Without loss of generality, we may assume that $0<r_{2} \leq r_{1}$ and omit the case $0<$ $r_{1}<r_{2}$ due to their similarity. For $0<r_{2} \leq r_{1}$, there are $k_{1}, k_{2} \in \mathbf{Z}$ such that $2^{k_{1}-1}<r_{1} \leq 2^{k_{1}}$ and $2^{k_{2}-1}<r_{2} \leq 2^{k_{2}}$. Then $k_{2} \leq k_{1}$ and

$$
\left(k_{1}-k_{2}-1\right) \ln 2<\ln \frac{r_{1}}{r_{2}}<\left(k_{1}-k_{2}+1\right) \ln 2 .
$$

Thus, we have

$$
\begin{aligned}
& \left(\frac{1}{\left|B\left(x, r_{1}\right)\right|} \int_{B\left(x, r_{1}\right)}\left|f(y)-f_{B\left(x, r_{2}\right)}\right|^{p} \omega(y)^{1-p} d y\right)^{\frac{1}{p}} \\
& \leq\left(\frac{1}{\left|B\left(x, r_{1}\right)\right|} \int_{B\left(x, r_{1}\right)}\left|f(y)-f_{B\left(x, 2^{\left.k_{1}\right)}\right.}\right|^{p} \omega(y)^{1-p} d y\right)^{\frac{1}{p}} \\
& \quad+\left|f_{B\left(x, r_{2}\right)}-f_{B\left(x, 2^{\left.k_{1}\right)}\right.}\right|\left(\frac{1}{\left|B\left(x, r_{1}\right)\right|} \int_{B\left(x, r_{1}\right)} \omega(y)^{1-p} d y\right)^{\frac{1}{p}}
\end{aligned}
$$


Lin et al. Journal of Inequalities and Applications ( 2015) 2015:338

Page 8 of 19

$$
\begin{aligned}
\leq & \left(\frac{2^{n}}{\left|B\left(x, 2^{k_{1}}\right)\right|} \int_{B\left(x, 2^{\left.k_{1}\right)}\right.}\left|f(y)-f_{B\left(x, 2^{\left.k_{1}\right)}\right.}\right|^{p} \omega(y)^{1-p} d y\right)^{\frac{1}{p}} \\
& +\left(\left|f_{B\left(x, r_{2}\right)}-f_{B\left(x, 2^{\left.k_{2}\right)}\right.}\right|+\sum_{j=k_{2}}^{k_{1}-1}\left|f_{B\left(x, 2^{j+1}\right)}-f_{B\left(x, 2^{j}\right)}\right|\right) \\
& \times\left(\frac{1}{\left|B\left(x, r_{1}\right)\right|} \int_{B\left(x, r_{1}\right)} \omega(y)^{1-p} d y\right)^{\frac{1}{p}} \\
\leq & C \mid f \|_{\operatorname{Lip}_{\beta_{0}}(\omega)} \omega(x)^{\frac{1}{p}} \omega\left(B\left(x, 2^{k_{1}}\right)\right)^{\beta_{0} / n} \\
& +\left(\frac{1}{\left|B\left(x, r_{2}\right)\right|} \int_{B\left(x, r_{2}\right)}\left|f(y)-f_{B\left(x, 2^{\left.k_{2}\right)}\right.}\right| d y\right. \\
& \left.+\sum_{j=k_{2}}^{k_{1}-1} \frac{1}{\left|B\left(x, 2^{j}\right)\right|} \int_{B\left(x, 2^{j}\right)}\left|f(y)-f_{B\left(x, 2^{j+1}\right)}\right| d y\right) \\
& \times\left(\frac{1}{\left|B\left(x, r_{1}\right)\right|} \int_{B\left(x, r_{1}\right)} \omega(y)^{1-p} d y\right)^{\frac{1}{p}} .
\end{aligned}
$$

Write

$$
\begin{aligned}
& \frac{1}{\left|B\left(x, r_{2}\right)\right|} \int_{B\left(x, r_{2}\right)}\left|f(y)-f_{B\left(x, 2^{k_{2}}\right)}\right| d y \\
& \leq \frac{2^{n}}{\left|B\left(x, 2^{k_{2}}\right)\right|}\left(\int_{B\left(x, 2^{k_{2}}\right)}\left|f(y)-f_{B\left(x, 2^{k_{2}}\right)}\right|^{p} \omega(y)^{1-p} d y\right)^{\frac{1}{p}} \\
& \quad \times\left(\int_{B\left(x, 2^{k_{2}}\right)} \omega(y) d y\right)^{\frac{1}{p^{\prime}}} \\
& \leq 2^{n} \frac{\omega\left(B\left(x, 2^{k_{2}}\right)\right)}{\left|B\left(x, 2^{k_{2}}\right)\right|} \\
& \quad \times\left(\frac{1}{\omega\left(B\left(x, 2^{k_{2}}\right)\right)} \int_{B\left(x, 2^{k_{2}}\right)}\left|f(y)-f_{B\left(x, 2^{k_{2}}\right)}\right|^{p} \omega(y)^{1-p} d y\right)^{\frac{1}{p}} \\
& \leq 2^{n}\|f\|_{\operatorname{Lip}_{\beta_{0}}(\omega)} \omega(x) \omega\left(B\left(x, 2^{k_{2}}\right)\right)^{\beta_{0} / n}
\end{aligned}
$$

and

$$
\begin{aligned}
& \frac{1}{\left|B\left(x, 2^{j}\right)\right|} \int_{B\left(x, 2^{j}\right)}\left|f(y)-f_{B\left(x, 2^{j+1}\right)}\right| d y \\
& \leq \frac{2^{n}}{\left|B\left(x, 2^{j+1}\right)\right|}\left(\int_{B\left(x, 2^{j+1}\right)}\left|f(y)-f_{B\left(x, 2^{j+1}\right)}\right|^{p} \omega(y)^{1-p} d y\right)^{\frac{1}{p}} \\
& \quad \times\left(\int_{B\left(x, 2^{j+1}\right)} \omega(y) d y\right)^{\frac{1}{p^{\prime}}} \\
& \leq 2^{n} \frac{\omega\left(B\left(x, 2^{j+1}\right)\right)}{\left|B\left(x, 2^{j+1}\right)\right|} \\
& \quad \times\left(\frac{1}{\omega\left(B\left(x, 2^{j+1}\right)\right)} \int_{B\left(x, 2^{j+1}\right)}\left|f(y)-f_{B\left(x, 2^{j+1}\right)}\right|^{p} \omega(y)^{1-p} d y\right)^{\frac{1}{p}} \\
& \leq 2^{n}\|f\|_{\operatorname{Lip}_{\beta_{0}}(\omega)} \omega(x) \omega\left(B\left(x, 2^{j+1}\right)\right)^{\beta_{0} / n} .
\end{aligned}
$$


If $1<p<\infty$, then it follows from $\omega \in A_{1} \subset A_{p^{\prime}}$ that

$$
\begin{aligned}
& \left(\frac{1}{\left|B\left(x, r_{1}\right)\right|} \int_{B\left(x, r_{1}\right)} \omega(y)^{1-p} d y\right)^{\frac{1}{p}} \\
& \quad \leq C\left(\frac{1}{\left|B\left(x, r_{1}\right)\right|} \int_{B\left(x, r_{1}\right)} \omega(y) d y\right)^{-\frac{1}{p\left(p^{\prime}-1\right)}} \\
& \quad=C\left(\frac{\omega\left(B\left(x, r_{1}\right)\right)}{\left|B\left(x, r_{1}\right)\right|}\right)^{-\frac{1}{p^{\prime}}}
\end{aligned}
$$

If $p=1$, then the above estimate holds obviously.

Thus

$$
\begin{aligned}
& \left(\frac{1}{\left|B\left(x, r_{1}\right)\right|} \int_{B\left(x, r_{1}\right)}\left|f(y)-f_{B\left(x, r_{2}\right)}\right|^{p} \omega(y)^{1-p} d y\right)^{\frac{1}{p}} \\
& \leq C\|f\|_{\operatorname{Lip}_{\beta_{0}}(\omega)} \omega(x)^{\frac{1}{p}} \omega\left(B\left(x, 2^{k_{1}}\right)\right)^{\beta_{0} / n} \\
& +C\left(\|f\|_{\operatorname{Lip}_{\beta_{0}}(\omega)} \omega(x) \omega\left(B\left(x, 2^{k_{2}}\right)\right)^{\beta_{0} / n}\right. \\
& \left.+\sum_{j=k_{2}}^{k_{1}-1}\|f\|_{\operatorname{Lip}_{\beta_{0}}(\omega)} \omega(x) \omega\left(B\left(x, 2^{j+1}\right)\right)^{\beta_{0} / n}\right)\left(\frac{\omega\left(B\left(x, r_{1}\right)\right)}{\left|B\left(x, r_{1}\right)\right|}\right)^{-\frac{1}{p^{\prime}}} \\
& \leq C\|f\|_{\operatorname{Lip}_{\beta_{0}}(\omega)} \omega(x) \omega\left(B\left(x, 2^{k_{1}}\right)\right)^{\beta_{0} / n}\left(\frac{\omega\left(B\left(x, r_{1}\right)\right)}{\left|B\left(x, r_{1}\right)\right|}\right)^{-\frac{1}{p^{\prime}}} \\
& +C\left(k_{1}-k_{2}+1\right)\|f\|_{\operatorname{Lip}_{\beta_{0}}(\omega)} \omega(x) \omega\left(B\left(x, 2^{k_{1}}\right)\right)^{\beta_{0} / n} \\
& \times\left(\frac{\omega\left(B\left(x, r_{1}\right)\right)}{\left|B\left(x, r_{1}\right)\right|}\right)^{-\frac{1}{p^{\prime}}} \\
& \leq C\|f\|_{\operatorname{Lip} \beta_{0}} \omega(x)\left(1+\left|\ln \frac{r_{2}}{r_{1}}\right|\right)\left(\frac{\omega\left(B\left(x, r_{1}\right)\right)}{\left|B\left(x, r_{1}\right)\right|}\right)^{-\frac{1}{p^{\prime}}} \omega\left(B\left(x, r_{1}\right)\right)^{\beta_{0} / n} .
\end{aligned}
$$

This completes the proof of Lemma 3.11.

Lemma 3.12 Let $T$ be a strongly singular Calderón-Zygmund operator, $\alpha, \beta, \delta$ be given as in Definition 1.1 and $\frac{n(1-\alpha)}{2}<\beta<\frac{n}{2}$. Let $0<\beta_{0}<1,0<t<1, \frac{n(1-\alpha)+2 \beta}{2 \beta}<s<\infty, \omega \in A_{1} \cap R H_{r}$ with $r>\frac{(n(1-\alpha)+2 \beta)(s-1)}{2 \beta s-n(1-\alpha)-2 \beta}$, and $b \in \operatorname{Lip}_{\beta_{0}}(\omega)$, then we have, for a.e. $x \in \mathbf{R}^{n}$,

$$
M_{t}^{\sharp}([b, T] f)(x) \leq C\|b\|_{\operatorname{Lip}_{\beta_{0}}(\omega)}\left(\omega(x) M_{\beta_{0}, s, \omega}(T f)(x)+\omega(x) M_{\beta_{0}, s, \omega}(f)(x)\right) .
$$

Proof For any ball $B=B\left(x, r_{B}\right)$ with the center $x$ and radius $r_{B}$, there are two cases.

Case 1: $r_{B}>1$.

We decompose $f=f_{1}+f_{2}$, where $f_{1}=f \chi_{2 B}$ and $\chi_{2 B}$ denotes the characteristic function of $2 B$. Observe that

$$
[b, T](f)(y)=\left(b(y)-b_{2 B}\right) T(f)(y)-T\left(\left(b-b_{2 B}\right) f_{1}\right)(y)-T\left(\left(b-b_{2 B}\right) f_{2}\right)(y) .
$$


Since $0<t<1$, we have

$$
\begin{aligned}
\left(\left.\frac{1}{|B|} \int_{B}||[b, T](f)(y)\right|^{t}-\left|T\left(\left(b-b_{2 B}\right) f_{2}\right)(x)\right|^{t} \mid d y\right)^{1 / t} \\
\leq\left(\frac{1}{|B|} \int_{B}\left|[b, T](f)(y)+T\left(\left(b-b_{2 B}\right) f_{2}\right)(x)\right|^{t} d y\right)^{1 / t} \\
\leq C\left(\frac{1}{|B|} \int_{B}\left|\left(b(y)-b_{2 B}\right) T(f)(y)\right|^{t} d y\right)^{1 / t} \\
\quad+C\left(\frac{1}{|B|} \int_{B}\left|T\left(\left(b-b_{2 B}\right) f_{1}\right)(y)\right|^{t} d y\right)^{1 / t} \\
\quad+C\left(\frac{1}{|B|} \int_{B}\left|T\left(\left(b-b_{2 B}\right) f_{2}\right)(y)-T\left(\left(b-b_{2 B}\right) f_{2}\right)(x)\right|^{t} d y\right)^{1 / t} \\
:=I_{1}+I_{2}+I_{3} .
\end{aligned}
$$

Since $\omega \in A_{1}$, by Hölder's inequality and Lemma 3.10, we have

$$
\begin{aligned}
I_{1} \leq & \frac{C}{|B|} \int_{B}\left|\left(b(y)-b_{2 B}\right) T(f)(y)\right| d y \\
\leq & \frac{C}{|B|}\left(\int_{B}\left|b(y)-b_{2 B}\right|^{s^{\prime}} \omega(y)^{1-s^{\prime}} d y\right)^{1 / s^{\prime}}\left(\int_{B}|T f(y)|^{s} \omega(y) d y\right)^{1 / s} \\
\leq & \frac{C}{\omega(2 B)^{\beta_{0} / n}}\left(\frac{1}{\omega(2 B)} \int_{2 B}\left|b(y)-b_{2 B}\right|^{s^{\prime}} \omega(y)^{1-s^{\prime}} d y\right)^{1 / s^{\prime}} \\
& \times\left(\frac{C}{\omega(B)^{1-\beta_{0} s / n}} \int_{B}|T f(y)|^{s} \omega(y) d y\right)^{1 / s} \frac{\omega(B)}{|B|} \\
\leq & C\|b\|_{\operatorname{Lip}_{\beta_{0}}(\omega)} M_{\beta_{0}, s, \omega}(T f)(x) \omega(x) .
\end{aligned}
$$

It follows from Kolmogorov's inequality [22], Lemma 3.1, Lemma 3.4, Hölder's inequality and Lemma 3.10 that

$$
\begin{aligned}
I_{2} \leq & \frac{C}{|B|} \int_{2 B}\left|\left(b(y)-b_{2 B}\right) f(y)\right| d y \\
\leq & \frac{C}{|B|}\left(\int_{2 B}\left|b(y)-b_{2 B}\right|^{s^{\prime}} \omega(y)^{1-s^{\prime}} d y\right)^{1 / s^{\prime}}\left(\int_{2 B}|f(y)|^{s} \omega(y) d y\right)^{1 / s} \\
\leq & \frac{C}{\omega(2 B)^{\beta_{0} / n}}\left(\frac{1}{\omega(2 B)} \int_{2 B}\left|b(y)-b_{2 B}\right|^{s^{\prime}} \omega(y)^{1-s^{\prime}} d y\right)^{1 / s^{\prime}} \\
& \times\left(\frac{1}{\omega(2 B)^{1-\beta_{0} s / n}} \int_{2 B}|f(y)|^{s} \omega(y) d y\right)^{1 / s} \frac{\omega(2 B)}{|2 B|} \\
\leq & C\|b\|_{\operatorname{Lip} \beta_{0}(\omega)} M_{\beta_{0}, s, \omega}(f)(x) \omega(x) .
\end{aligned}
$$

Since $r_{B}>1$ and $2|y-x|^{\alpha} \leq|z-x|$ for any $y \in B, z \in(2 B)^{c}$, by (2) of Definition 1.1, we can get

$$
\begin{aligned}
I_{3} & \leq \frac{C}{|B|} \int_{B}\left|T\left(\left(b-b_{2 B}\right) f_{2}\right)(y)-T\left(\left(b-b_{2 B}\right) f_{2}\right)(x)\right| d y \\
& \leq \frac{C}{|B|} \int_{B} \int_{(2 B)^{c}}|K(y, z)-K(x, z)|\left|b(z)-b_{2 B}\right||f(z)| d z d y
\end{aligned}
$$




$$
\begin{aligned}
& \leq C \sum_{j=1}^{\infty} \frac{1}{|B|} \int_{B} \int_{2^{j+1} B \backslash 2 j_{B}} \frac{|y-x|^{\delta}}{|z-x|^{n+\frac{\delta}{\alpha}}}\left|b(z)-b_{2 B}\right||f(z)| d z d y \\
& \leq C r_{B}^{\delta-\frac{\delta}{\alpha}} \sum_{j=1}^{\infty}\left(2^{j}\right)^{-\frac{\delta}{\alpha}} \frac{1}{\left|2^{j+1} B\right|} \int_{2^{j+1} B}\left|b(z)-b_{2 B}\right||f(z)| d z .
\end{aligned}
$$

By Hölder's inequality and Lemma 3.11, we have

$$
\begin{aligned}
I_{3} \leq & C \sum_{j=1}^{\infty}\left(2^{j}\right)^{-\frac{\delta}{\alpha}} \frac{1}{\left|2^{j+1} B\right|}\left(\int_{2^{j+1} B}\left|b(z)-b_{2 B}\right|^{s^{\prime}} \omega(z)^{1-s^{\prime}} d z\right)^{1 / s^{\prime}} \\
& \times\left(\int_{2^{j+1} B}|f(z)|^{s} \omega(z) d z\right)^{1 / s} \\
\leq & C \sum_{j=1}^{\infty}\left(2^{j}\right)^{-\frac{\delta}{\alpha}} \frac{1}{\left|2^{j+1} B\right|}\left|2^{j+1} B\right|^{\frac{1}{s}}\left(\int_{2^{j+1} B}|f(z)|^{s} \omega(z) d z\right)^{1 / s} \\
& \times\|b\|_{\operatorname{Lip}_{\beta_{0}}(\omega)} \omega(x)\left(1+\left|\ln \frac{2}{2^{j+1}}\right|\right)\left(\frac{\omega\left(2^{j+1} B\right)}{\left|2^{j+1} B\right|}\right)^{-\frac{1}{s}} \omega\left(2^{j+1} B\right)^{\beta_{0} / n} \\
\leq & C\|b\|_{\operatorname{Lip}_{\beta_{0}}(\omega)} \omega(x) \sum_{j=1}^{\infty} j\left(2^{j}\right)^{-\frac{\delta}{\alpha}} \frac{1}{\left|2^{j+1} B\right|}\left|2^{j+1} B\right|^{\frac{1}{s^{\prime}}}\left(\frac{\omega\left(2^{j+1} B\right)}{\left|2^{j+1} B\right|}\right)^{-\frac{1}{s}} \\
& \times \omega\left(2^{j+1} B\right)^{1 / s}\left(\frac{1}{\omega\left(2^{j+1} B\right)^{1-\beta_{0} s / n}} \int_{2^{j+1} B}|f(z)|^{s} \omega(z) d z\right)^{1 / s} \\
\leq & C\|b\|_{\operatorname{Lip}_{\beta_{0}}(\omega)} \omega(x) M_{\beta_{0}, s, \omega}(f)(x) \sum_{j=1}^{\infty} j\left(2^{j}\right)^{-\frac{\delta}{\alpha}} \\
\leq & C\|b\|_{\operatorname{Lip}_{\beta_{0}}(\omega) \omega(x) M_{\beta_{0}, s, \omega}(f)(x) .}
\end{aligned}
$$

Case 2: $0<r_{B} \leq 1$.

Since $r>\frac{(n(1-\alpha)+2 \beta)(s-1)}{2 \beta s-n(1-\alpha)-2 \beta}$, then $\frac{n(1-\alpha)+2 \beta}{2 \beta}<\frac{r s}{s+r-1}$. There exists $s_{0}$ such that $\frac{n(1-\alpha)+2 \beta}{2 \beta}<s_{0}<$ $\frac{r s}{s+r-1}$. For this index $s_{0}$, there exists $l_{0}$ such that $T$ is bounded from $L^{s_{0}}$ to $L^{l_{0}}$ and $0<\frac{s_{0}}{l_{0}}<\alpha$. Then we can take $\theta$ satisfying $0<\frac{s_{0}}{l_{0}}<\theta<\alpha$. Let $\tilde{B}=B\left(x, r_{B}^{\theta}\right)$. Write $f=f_{3}+f_{4}$, where $f_{3}=f \chi_{2 \tilde{B}}$, then

$$
[b, T](f)(y)=\left(b(y)-b_{2 B}\right) T(f)(y)-T\left(\left(b-b_{2 B}\right) f_{3}\right)(y)-T\left(\left(b-b_{2 B}\right) f_{4}\right)(y) .
$$

Since $0<t<1$, we have

$$
\begin{aligned}
& \left(\left.\frac{1}{|B|} \int_{B}||[b, T](f)(y)\right|^{t}-\left|T\left(\left(b-b_{2 B}\right) f_{4}\right)(x)\right|^{t} \mid d y\right)^{1 / t} \\
& \leq\left(\frac{1}{|B|} \int_{B}\left|[b, T](f)(y)+T\left(\left(b-b_{2 B}\right) f_{4}\right)(x)\right|^{t} d y\right)^{1 / t} \\
& \leq C\left(\frac{1}{|B|} \int_{B}\left|\left(b(y)-b_{2 B}\right) T(f)(y)\right|^{t} d y\right)^{1 / t} \\
& \quad+C\left(\frac{1}{|B|} \int_{B}\left|T\left(\left(b-b_{2 B}\right) f_{3}\right)(y)\right|^{t} d y\right)^{1 / t}
\end{aligned}
$$




$$
\begin{aligned}
& +C\left(\frac{1}{|B|} \int_{B}\left|T\left(\left(b-b_{2 B}\right) f_{4}\right)(y)-T\left(\left(b-b_{2 B}\right) f_{4}\right)(x)\right|^{t} d y\right)^{1 / t} \\
:= & I I_{1}+I I_{2}+I I_{3} .
\end{aligned}
$$

Similarly to estimate $I_{1}$, we get

$$
I I_{1} \leq C\|b\|_{\operatorname{Lip}_{\beta_{0}}(\omega)} M_{\beta_{0}, s, \omega}(T f)(x) \omega(x)
$$

Since $1<s_{0}<s<\infty$, there exists $l(1<l<\infty)$ such that $\frac{1}{s_{0}}=\frac{1}{s}+\frac{1}{l}$. It follows from Hölder's inequality and the $\left(L^{s_{0}}, L^{l_{0}}\right)$-boundedness of $T$ that

$$
\begin{aligned}
I I_{2} & \leq C\left(\frac{1}{|B|} \int_{B}\left|T\left(\left(b-b_{2 B}\right) f_{3}\right)(y)\right|^{l_{0}} d y\right)^{\frac{1}{l_{0}}} \\
& \leq C|B|^{-\frac{1}{l_{0}}}\left(\int_{2 \tilde{B}}\left|b(y)-b_{2 B}\right|^{s_{0}}|f(y)|^{s_{0}} d y\right)^{\frac{1}{s_{0}}} \\
& \leq C|B|^{-\frac{1}{l_{0}}}\left(\int_{2 \tilde{B}}\left|b(y)-b_{2 B}\right|^{l} \omega(y)^{-\frac{l}{s}} d y\right)^{\frac{1}{l}}\left(\int_{2 \tilde{B}}|f(y)|^{s} \omega(y) d y\right)^{\frac{1}{s}} \\
& \leq C M_{\beta_{0}, s, \omega}(f)(x) \omega(2 \tilde{B})^{\frac{1}{s}-\frac{\beta_{0}}{n}}|B|^{-\frac{1}{l_{0}}}\left(\int_{2 \tilde{B}}\left|b(y)-b_{2 B}\right|^{l} \omega(y)^{-\frac{l}{s}} d y\right)^{\frac{1}{l}} .
\end{aligned}
$$

Denote $p_{0}=\frac{(r-1)\left(s-s_{0}\right)}{s\left(s_{0}-1\right)}$. The fact $s_{0}<\frac{r s}{s+r-1}$ implies $1<p_{0}<\infty$. Then we get $p_{0}^{\prime}=\frac{(r-1)\left(s-s_{0}\right)}{r s-(s+r-1) s_{0}}$ and $1<p_{0}^{\prime}<\infty$. By Hölder's inequality, Lemma 3.11, and noticing that $r=\frac{l p_{0}}{s^{\prime}}-\frac{p_{0}}{p_{0}^{\prime}}$, we have

$$
\begin{aligned}
I I_{2} \leq & C M_{\beta_{0}, s, \omega}(f)(x) \omega(2 \tilde{B})^{\frac{1}{s}-\frac{\beta_{0}}{n}}|B|^{-\frac{1}{l_{0}}}\left(\int_{2 \tilde{B}} \omega(y)^{\frac{l p_{0}}{s^{\prime}} \frac{p_{0}}{p_{0}^{\prime}}} d y\right)^{\frac{1}{l_{0}}} \\
& \times\left(\int_{2 \tilde{B}}\left|b(y)-b_{2 B}\right|^{l p_{0}^{\prime}} \omega(y)^{1-l p_{0}^{\prime}} d y\right)^{\frac{1}{l p_{0}^{\prime}}} \\
\leq & C M_{\beta_{0}, s, \omega}(f)(x) \omega(2 \tilde{B})^{\frac{1}{s}-\frac{\beta_{0}}{n}}|B|^{-\frac{1}{l_{0}}}|2 \tilde{B}|^{\frac{1}{l}}\|b\|_{\operatorname{Lip}_{\beta_{0}}(\omega) \omega(x)} \\
& \times\left(1+\mid \ln \frac{r_{B}^{\theta}}{r_{B} \mid}\right)\left(\frac{\omega(2 \tilde{B})}{|2 \tilde{B}|}\right)^{-\frac{1}{\left(l p_{0}^{\prime}\right)^{\prime}}}\left(\frac{\omega(2 \tilde{B})}{|2 \tilde{B}|}\right)^{\frac{r}{l_{0}}} \omega(2 \tilde{B})^{\frac{\beta_{0}}{n}} \\
\leq & C\|b\|_{\operatorname{Lip}_{\beta_{0}}(\omega) \omega(x) M_{\beta_{0}, s, \omega}(f)(x) \omega(2 \tilde{B})^{\frac{1}{s}}|B|^{-\frac{1}{l_{0}}}|2 \tilde{B}|^{\frac{1}{l}}} \\
& \times\left(1+(1-\theta) \ln \frac{1}{r_{B}}\right)\left(\frac{\omega(2 \tilde{B})}{|2 \tilde{B}|}\right)^{-\frac{1}{s}} \cdot
\end{aligned}
$$

Denote $\varepsilon_{1}=n\left(\frac{\theta}{s_{0}}-\frac{1}{l_{0}}\right)$. The inequality $0<\frac{s_{0}}{l_{0}}<\theta$ implies that $\varepsilon_{1}>0$. By Lemma 3.7, we have

$$
\begin{aligned}
I I_{2} & \leq C\|b\|_{\operatorname{Lip}_{\beta_{0}}(\omega)} \omega(x) M_{\beta_{0}, s, \omega}(f)(x)|B|^{-\frac{1}{l_{0}}}|2 \tilde{B}|^{\frac{1}{l}+\frac{1}{s}}\left(1+\frac{1}{\varepsilon_{1}} r_{B}^{-\varepsilon_{1}}\right) \\
& \leq C\|b\|_{\operatorname{Lip}_{\beta_{0}}(\omega)} \omega(x) M_{\beta_{0}, s, \omega}(f)(x) r_{B}^{n\left(\frac{\theta}{s_{0}}-\frac{1}{l_{0}}\right)-\varepsilon_{1}} \\
& =C\|b\|_{\operatorname{Lip}_{\beta_{0}}(\omega)} \omega(x) M_{\beta_{0}, s, \omega}(f)(x) .
\end{aligned}
$$


Let $\varepsilon_{2}=\frac{\delta}{\alpha}(\alpha-\theta)$. It follows from $\theta<\alpha$ that $\varepsilon_{2}>0$. For any $y \in B$ and $z \in(2 \tilde{B})^{c}$, there is $2|y-x|^{\alpha} \leq 2 r_{B}^{\alpha} \leq 2 r_{B}^{\theta} \leq|z-x|$ since $0<r_{B} \leq 1$. Applying (2) of Definition 1.1, Hölder's inequality, Lemma 3.7 and Lemma 3.11, we get

$$
\begin{aligned}
& I I_{3} \leq \frac{C}{|B|} \int_{B}\left|T\left(\left(b-b_{2 B}\right) f_{4}\right)(y)-T\left(\left(b-b_{2 B}\right) f_{4}\right)(x)\right| d y \\
& \leq \frac{C}{|B|} \int_{B} \int_{(2 \tilde{B})^{c}}|K(y, z)-K(x, z)|\left|b(z)-b_{2 B}\right||f(z)| d z d y \\
& \leq C \sum_{j=1}^{\infty} \frac{1}{|B|} \int_{B} \int_{2^{j+1} \tilde{B} \backslash 2 j \tilde{B}} \frac{|y-x|^{\delta}}{|z-x|^{n+\frac{\delta}{\alpha}}}\left|b(z)-b_{2 B}\right||f(z)| d z d y \\
& \leq C r_{B}^{\delta-\frac{\theta \delta}{\alpha}} \sum_{j=1}^{\infty}\left(2^{j}\right)^{-\frac{\delta}{\alpha}} \frac{1}{\left|2^{j+1} \tilde{B}\right|}\left(\int_{2^{j+1} \tilde{B}}\left|b(z)-b_{2 B}\right|^{s^{\prime}} \omega(y)^{1-s^{\prime}} d y\right)^{\frac{1}{s^{\prime}}} \\
& \times\left(\int_{2^{+1} \tilde{B}}|f(z)|^{s} \omega(y) d y\right)^{\frac{1}{s}} \\
& \leq C r_{B}^{\delta-\frac{\theta \delta}{\alpha}} \sum_{j=1}^{\infty}\left(2^{j}\right)^{-\frac{\delta}{\alpha}} \frac{1}{\left|2^{j+1} \tilde{B}\right|}\left|2^{j+1} \tilde{B}\right|^{\frac{1}{s^{\prime}}}\|b\|_{\operatorname{Lip}_{\beta_{0}}(\omega)} \omega(x) \omega\left(2^{j+1} \tilde{B}\right)^{\frac{\beta_{0}}{n}} \\
& \times\left(1+\left|\ln \frac{2 r_{B}}{2^{j+1} r_{B}^{\theta}}\right|\right)\left(\frac{\omega\left(2^{j+1} \tilde{B}\right)}{\left|2^{j+1} \tilde{B}\right|}\right)^{-\frac{1}{s}} M_{\beta_{0}, s, \omega}(f)(x) \omega\left(2^{j+1} \tilde{B}\right)^{\frac{1}{s}-\frac{\beta_{0}}{n}} \\
& \leq C r_{B}^{\delta-\frac{\theta \delta}{\alpha}} \sum_{j=1}^{\infty}\left(2^{j}\right)^{-\frac{\delta}{\alpha}}\|b\|_{\operatorname{Lip}_{\beta_{0}}(\omega)} \omega(x) M_{\beta_{0}, s, \omega}(f)(x)\left(j+(1-\theta) \ln \frac{1}{r_{B}}\right) \\
& \leq C\|b\|_{\operatorname{Lip}_{\beta_{0}}(\omega)} \omega(x) M_{\beta_{0}, s, \omega}(f)(x) r_{B}^{\delta-\frac{\theta \delta}{\alpha}} \sum_{j=1}^{\infty}\left(2^{j}\right)^{-\frac{\delta}{\alpha}}\left(j+\frac{1}{\varepsilon_{2}} r_{B}^{-\varepsilon_{2}}\right) \\
& \leq C\|b\|_{\operatorname{Lip}_{\beta_{0}}(\omega)} \omega(x) M_{\beta_{0}, s, \omega}(f)(x) r_{B}^{\frac{\delta}{\alpha}(\alpha-\theta)-\varepsilon_{2}} \sum_{j=1}^{\infty} j\left(2^{j}\right)^{-\frac{\delta}{\alpha}} \\
& \leq C\|b\|_{\operatorname{Lip}_{\beta_{0}}(\omega)} \omega(x) M_{\beta_{0}, s, \omega}(f)(x) .
\end{aligned}
$$

Putting the estimates in both cases together, we have

$$
\begin{aligned}
M_{t}^{\sharp}([b, T] f)(x) & \sim \sup _{r_{B}>0} \inf _{a \in \mathbf{C}}\left(\frac{1}{\left|B\left(x, r_{B}\right)\right|} \int_{B\left(x, r_{B}\right)} \|\left.[b, T](f)(y)\right|^{t}-a \mid d y\right)^{\frac{1}{t}} \\
& \leq C\|b\|_{\operatorname{Lip}_{\beta_{0}}(\omega)}\left(\omega(x) M_{\beta_{0}, s, \omega}(T f)(x)+\omega(x) M_{\beta_{0}, s, \omega}(f)(x)\right) .
\end{aligned}
$$

This completes the proof of Lemma 3.12.

Lemma 3.13 Let $T$ be a generalized Calderón-Zygmund operator, $q^{\prime}$ be the same as in Definition 1.2 and the sequence $\left\{j C_{j}\right\} \in l^{1}$. Let $0<\delta<1,0<\beta_{0}<1, \omega \in A_{1}, r_{\omega}>q^{\prime}$, and $b \in \operatorname{Lip}_{\beta_{0}}(\omega)$, then, for all $r>\frac{r_{\omega}-1}{r_{\omega}-q^{\prime}} q^{\prime}$, we have

$$
\begin{aligned}
M_{\delta}^{\sharp}([b, T] f)(x) \leq & C\|b\|_{\operatorname{Lip}_{\beta_{0}}(\omega)}\left(\omega(x) M_{\beta_{0}, r, \omega}(T f)(x)\right. \\
& \left.+\omega(x) M_{\beta_{0}, r, \omega}(f)(x)+\omega(x)^{1+\frac{\beta_{0}}{n}} M_{\beta_{0}, r}(f)(x)\right),
\end{aligned}
$$

a.e. $x \in \mathbf{R}^{n}$. 
Proof For any ball $B=B\left(x_{0}, r_{B}\right)$ which contains $x$, we decompose $f=f_{1}+f_{2}$, where $f_{1}=f_{\chi_{2 B} B}$. Observe that

$$
[b, T] f(y)=\left(b(y)-b_{2 B}\right) T f(y)-T\left(\left(b-b_{2 B}\right) f\right)(y) .
$$

Since $0<\delta<1$, we have

$$
\begin{aligned}
\left(\left.\frac{1}{|B|} \int_{B}||[b, T] f(y)\right|^{\delta}-\left|T\left(\left(b-b_{2 B}\right) f_{2}\right)\left(x_{0}\right)\right|^{\delta} \mid d y\right)^{1 / \delta} \\
\leq\left(\frac{1}{|B|} \int_{B}\left|[b, T] f(y)+T\left(\left(b-b_{2 B}\right) f_{2}\right)\left(x_{0}\right)\right|^{\delta} d y\right)^{1 / \delta} \\
\leq C\left(\frac{1}{|B|} \int_{B}\left|\left(b(y)-b_{2 B}\right) T f(y)\right|^{\delta} d y\right)^{1 / \delta} \\
\quad+C\left(\frac{1}{|B|} \int_{B}\left|T\left(\left(b-b_{2 B}\right) f_{1}\right)(y)\right|^{\delta} d y\right)^{1 / \delta} \\
\quad+C\left(\frac{1}{|B|} \int_{B}\left|T\left(\left(b-b_{2 B}\right) f_{2}\right)(y)-T\left(\left(b-b_{2 B}\right) f_{2}\right)\left(x_{0}\right)\right|^{\delta} d y\right)^{1 / \delta} \\
:=I+I I+I I I .
\end{aligned}
$$

Since $\omega \in A_{1}$, then it follows from Hölder's inequality and Lemma 3.10 that

$$
\begin{aligned}
I \leq & \frac{C}{|B|} \int_{B}\left|\left(b(y)-b_{2 B}\right) T f(y)\right| d y \\
\leq & \frac{C}{|B|}\left(\int_{B}\left|b(y)-b_{2 B}\right|^{r^{\prime}} \omega(y)^{1-r^{\prime}} d y\right)^{1 / r^{\prime}}\left(\int_{B}|T f(y)|^{r} \omega(y) d y\right)^{1 / r} \\
\leq & C \frac{1}{\omega(2 B)^{\beta_{0} / n}}\left(\frac{1}{\omega(2 B)} \int_{2 B}\left|b(y)-b_{2 B}\right|^{r^{\prime}} \omega(y)^{1-r^{\prime}} d y\right)^{1 / r^{\prime}} \\
& \times\left(\frac{1}{\omega(B)^{1-\frac{\beta_{0} r}{n}}} \int_{B}|T f(y)|^{r} \omega(y) d y\right)^{1 / r} \frac{\omega(B)}{|B|} \\
\leq & C\|b\|_{\operatorname{Lip}_{\beta_{0}}(\omega) \omega(x) M_{\beta_{0}, r, \omega}(T f)(x) .}
\end{aligned}
$$

By Kolmogorov's inequality, Lemma 3.2, Hölder's inequality and Lemma 3.10, we have

$$
\begin{aligned}
I I \leq & \frac{C}{|B|} \int_{2 B}\left|\left(b(y)-b_{2 B}\right) f(y)\right| d y \\
\leq & \frac{C}{|B|}\left(\int_{2 B}\left|b(y)-b_{2 B}\right|^{r^{\prime}} \omega(y)^{1-r^{\prime}} d y\right)^{1 / r^{\prime}}\left(\int_{2 B}|f(y)|^{r} \omega(y) d y\right)^{1 / r} \\
\leq & C \frac{1}{\omega(2 B)^{\beta_{0} / n}}\left(\frac{1}{\omega(2 B)} \int_{2 B}\left|b(y)-b_{2 B}\right|^{r^{\prime}} \omega(y)^{1-r^{\prime}} d y\right)^{1 / r^{\prime}} \\
& \times\left(\frac{1}{\omega(B)^{1-\frac{\beta_{0} r}{n}}} \int_{B}|f(y)|^{r} \omega(y) d y\right)^{1 / r} \frac{\omega(B)}{|B|} \\
\leq & C\|b\|_{\operatorname{Lip}_{\beta_{0}}(\omega) \omega(x) M_{\beta_{0}, r, \omega}(f)(x) .}
\end{aligned}
$$


For III, we have

$$
\begin{aligned}
I I I \leq & \frac{C}{|B|} \int_{B}\left|T\left(\left(b-b_{2 B}\right) f_{2}\right)(y)-T\left(\left(b-b_{2 B}\right) f_{2}\right)\left(x_{0}\right)\right| d y \\
\leq & \frac{C}{|B|} \int_{B} \int_{(2 B)^{c}}\left|K(y, z)-K\left(x_{0}, z\right)\right|\left|b(z)-b_{2 B}\right||f(z)| d z d y \\
\leq & C \sum_{j=1}^{\infty} \frac{1}{|B|} \int_{B} \int_{2^{j}\left|y-x_{0}\right| \leq\left|z-x_{0}\right|<2^{j+1}\left|y-x_{0}\right|}\left|K(y, z)-K\left(x_{0}, z\right)\right| \\
& \times\left|b(z)-b_{2 B}\right||f(z)| d z d y \quad\left|K(y, z)-K\left(x_{0}, z\right)\right| \\
\leq & C \sum_{j=1}^{\infty} \frac{1}{|B|} \int_{B} \int_{2^{j}\left|y-x_{0}\right| \leq\left|z-x_{0}\right|<2^{j+1}\left|y-x_{0}\right|}\left|K(y, z)-K\left(x_{0}, z\right)\right| \\
& \times\left|b(z)-b_{2^{j+1} B}\right||f(z)| d z d y \\
& +C \sum_{j=1}^{\infty} \frac{1}{|B|} \int_{B} \int_{2^{j}\left|y-x_{0}\right| \leq\left|z-x_{0}\right|<2^{j+1}\left|y-x_{0}\right|} \\
& \times\left|b_{2^{j+1} B}-b_{2 B}\right||f(z)| d z d y \\
:= & I I I_{1}+I I I_{2} .
\end{aligned}
$$

Since $r>\frac{r_{\omega}-1}{r_{\omega}-q^{\prime}} q^{\prime}>q^{\prime}$, there exists $1<l<\infty$ such that $\frac{1}{q}+\frac{1}{l}+\frac{1}{r}=1$. By Hölder's inequality for the three numbers $q, l$ and $r$, we can get

$$
\begin{aligned}
I I I_{1} \leq & C \sum_{j=1}^{\infty} \frac{1}{|B|} \int_{B}\left(\int_{2^{j}\left|y-x_{0}\right| \leq\left|z-x_{0}\right|<2^{j+1}\left|y-x_{0}\right|}\left|K(y, z)-K\left(x_{0}, z\right)\right|^{q} d z\right)^{1 / q} \\
& \times\left(\int_{2^{j}\left|y-x_{0}\right| \leq\left|z-x_{0}\right|<2^{j+1}\left|y-x_{0}\right|}\left|b(z)-b_{2^{j+1} B}\right|^{l} \omega(z)^{\left(1 / r^{\prime}-1\right) l} d z\right)^{1 / l} \\
& \times\left(\int_{2^{j}\left|y-x_{0}\right| \leq\left|z-x_{0}\right|<2^{j+1}\left|y-x_{0}\right|}|f(z)|^{r} \omega(z) d z\right)^{1 / r} d y \\
\leq & C \sum_{j=1}^{\infty} \frac{1}{|B|} \int_{B} C_{j}\left(2^{j}\left|y-x_{0}\right|\right)^{-n / q^{\prime}} d y \\
& \times\left(\int_{2^{j+1} B}\left|b(z)-b_{2^{j+1} B}\right|^{l} \omega(z)^{\left(1 / r^{\prime}-1\right) l} d z\right)^{1 / l} \\
& \times\left(\int_{2^{j+1} B}|f(z)|^{r} \omega(z) d z\right)^{1 / r} .
\end{aligned}
$$

Denote $p_{0}=\frac{s-1}{\frac{q^{\prime}(r-1)}{r-q^{\prime}}-1}$. It follows from $r>\frac{r_{\omega}-1}{r_{\omega}-q^{\prime}} q^{\prime}$ that $r_{\omega}>\frac{r-1}{r-q^{\prime}} q^{\prime}$. Since $r_{\omega}=\sup \{s>1: \omega \in$ $\left.R H_{s}\right\}$, there exists $s$ such that $\omega \in R H_{s}$ and $s>\frac{r-1}{r-q^{\prime}} q^{\prime}$. We can get $1<p_{0}<\infty$ and $s=\frac{l p_{0}}{r^{\prime}}-\frac{p_{0}}{p_{0}^{\prime}}$. Applying Hölder's inequality for $p_{0}$ and $p_{0}^{\prime}$, we have

$$
\begin{aligned}
I I I_{1} \leq & C \sum_{j=1}^{\infty} \frac{1}{|B|} \int_{B} C_{j}\left(2^{j}\left|y-x_{0}\right|\right)^{-n / q^{\prime}} d y\left(\int_{2^{j+1} B}|f(z)|^{r} \omega(z) d z\right)^{1 / r} \\
& \times\left(\int_{2^{j+1} B}\left|b(z)-b_{2^{j+1} B}\right|^{l p_{0}^{\prime}} \omega(z)^{1-l p_{0}^{\prime}} d z\right)^{\frac{1}{l p_{0}^{\prime}}}
\end{aligned}
$$




$$
\begin{aligned}
& \times\left(\int_{2^{j+1} B} \omega(z)^{\frac{l p_{0}}{r^{\prime}}-\frac{p_{0}}{p_{0}^{\prime}}} d z\right)^{\frac{1}{l p_{0}}} \\
& \leq C \sum_{j=1}^{\infty} C_{j}\left(2^{j}\right)^{-n / q^{\prime}}|B|^{-1 / q^{\prime}} \omega\left(2^{j+1} B\right)^{\frac{1}{l_{0}^{\prime}}+\frac{1}{r}\left(1-\frac{\beta_{0} r}{n}\right)} \\
& \times\left(\int_{2^{j+1} B} \omega(z)^{\frac{l p_{0}}{r^{\prime}}-\frac{p_{0}}{p_{0}^{\prime}}} d z\right)^{\frac{1}{l p_{0}}} \\
& \times\left(\frac{1}{\omega\left(2^{j+1} B\right)^{1-\beta_{0} r / n}} \int_{2^{j+1} B}|f(z)|^{r} \omega(z) d z\right)^{\frac{1}{r}} \\
& \times\left(\frac{1}{\omega\left(2^{j+1} B\right)} \int_{2^{j+1} B}\left|b(z)-b_{2^{j+1} B}\right|^{l p_{0}^{\prime}} \omega(z)^{1-l p_{0}^{\prime}} d z\right)^{\frac{1}{l_{p}^{\prime}}} \\
& \leq C\|b\|_{\operatorname{Lip}_{\beta_{0}}(\omega)} M_{\beta_{0}, r, \omega}(f)(x) \sum_{j=1}^{\infty} C_{j}\left(2^{j}\right)^{-n / q^{\prime}}|B|^{-1 / q^{\prime}} \\
& \times\left[\left(\frac{1}{\left|2^{j+1} B\right|} \int_{2^{j+1} B} \omega(z)^{s} d z\right)^{1 / s}\right]^{\frac{s}{l p_{0}}} \omega\left(2^{j+1} B\right)^{\frac{1}{l_{0}^{\prime}}+\frac{1}{r}}\left|2^{j+1} B\right|^{\frac{1}{p_{0}}} \\
& \leq C\|b\|_{\operatorname{Lip}_{\beta_{0}}(\omega)} M_{\beta_{0}, r, \omega}(f)(x) \sum_{j=1}^{\infty} C_{j}\left(2^{j}\right)^{-n / q^{\prime}}|B|^{-1 / q^{\prime}} \\
& \times \frac{\omega\left(2^{j+1} B\right)^{\frac{1}{r^{\prime}}-\frac{1}{l p_{0}^{\prime}}+\frac{1}{l p_{0}^{\prime}}+\frac{1}{r}}}{\left|2^{j+1} B\right|^{\frac{1}{r^{\prime}}-\frac{1}{l p_{0}^{\prime}}-\frac{1}{l p_{0}}}} \\
& \leq C\|b\|_{\operatorname{Lip}_{\beta_{0}}(\omega)} M_{\beta_{0}, r, \omega}(f)(x) \omega(x) .
\end{aligned}
$$

Noticing that $\omega \in A_{1}$, it follows from Lemma 3.11 that

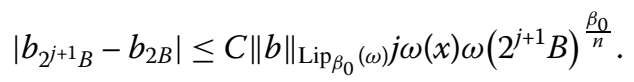

It follows from Hölder's inequality that

$$
\begin{aligned}
I I I_{2} \leq & C \sum_{j=1}^{\infty} \frac{1}{|B|} \int_{B}\left(\int_{2^{j}\left|y-x_{0}\right| \leq\left|z-x_{0}\right|<2^{j+1}\left|y-x_{0}\right|}\left|K(y, z)-K\left(x_{0}, z\right)\right|^{q} d z\right)^{1 / q} \\
& \times\left(\int_{2^{j+1} B}\left|b_{2^{j+1} B}-b_{2 B}\right|^{l} d z\right)^{1 / l}\left(\int_{2^{j+1} B}|f(z)|^{r} d z\right)^{1 / r} d y \\
\leq & C \sum_{j=1}^{\infty} C_{j}\left(2^{j}\right)^{-n / q^{\prime}} \frac{1}{|B|} \int_{B}\left|y-x_{0}\right|^{-n / q^{\prime}} d y\left|b_{2^{j+1} B}-b_{2 B}\right|\left|2^{j+1} B\right|^{1 / l} \\
& \times\left(\frac{1}{\left|2^{j+1} B\right|^{1-\frac{\beta_{0} r}{n}}} \int_{2^{j+1} B}|f(z)|^{r} d z\right)^{1 / r}\left|2^{j+1} B\right|^{\frac{1}{r}-\frac{\beta_{0}}{n}} \\
\leq & C\|b\|_{\operatorname{Lip}_{\beta_{0}}(\omega)} \omega(x) \sum_{j=1}^{\infty} j C_{j}\left(2^{j}\right)^{-n / q^{\prime}}|B|^{-1 / q^{\prime}} \\
& \times \omega\left(2^{j+1} B\right)^{\frac{\beta_{0}}{n}}\left|2^{j+1} B\right|^{\frac{1}{l}} M_{\beta_{0}, r}(f)(x)\left|2^{j+1} B\right|^{\frac{1}{r}-\frac{\beta_{0}}{n}} \\
\leq & C\|b\|_{\operatorname{Lip}_{\beta_{0}}(\omega)} M_{\beta_{0}, r}(f)(x) \omega(x)
\end{aligned}
$$




$$
\begin{aligned}
& \times \sum_{j=1}^{\infty} j C_{j}\left(2^{j}\right)^{-n / q^{\prime}}\left(2^{j+1}\right)^{n / q^{\prime}}\left|2^{j+1} B\right|^{\frac{1}{l^{+}+\frac{1}{r}-\frac{1}{q^{\prime}}} \omega(x)^{\frac{\beta_{0}}{n}}} \\
\leq & C\|b\|_{\operatorname{Lip}_{\beta_{0}}(\omega)} M_{\beta_{0}, r}(f)(x) \omega(x)^{1+\frac{\beta_{0}}{n}} .
\end{aligned}
$$

Putting the above estimates together and taking the supremum over all balls $B$ which contain $x$, we can get the desired result.

Lemma 3.14 Let $0<\beta_{0}<n, 1<p<n / \beta_{0}, 1 / s=1 / p-\beta_{0} / n$, and $\omega^{s / p} \in A_{1}$. Then, if $1<r<p$, $0<k<p / s, r_{\omega}>\frac{1-k}{p / s-k}$, we have

$$
\left\|M_{\beta_{0}, r}(f)\right\|_{L^{s, k s / p}\left(\omega^{s / p}, \omega\right)} \leq C\|f\|_{L^{p, k}(\omega)} .
$$

Proof Note that

$$
M_{\beta_{0}, r}(f)(x)=\left(M_{\beta_{0}, 1}\left(|f|^{r}\right)(x)\right)^{1 / r} .
$$

Let $\tilde{\beta}=\beta_{0} r, \tilde{s}=s / r$, and $\tilde{p}=p / r$. We have $0<\tilde{\beta}<n$ and $1<\tilde{p}<n / \tilde{\beta}$. Then $1 / \tilde{s}=1 / \tilde{p}-\tilde{\beta} / n$, $\omega^{\tilde{s} / \tilde{p}} \in A_{1}, 0<k<\tilde{p} / \tilde{s}$, and $r_{\omega}>\frac{1-k}{\tilde{p} / \tilde{s}-k}$. Using Lemma 3.8, we obtain

$$
\begin{aligned}
\left\|M_{\beta_{0}, r}(f)\right\|_{L^{s, k s} / p\left(\omega^{s / p, \omega)}\right.} & =\left\|M_{\tilde{\beta}, 1}\left(|f|^{r}\right)\right\|_{L^{\tilde{s}, k s} \tilde{s} \tilde{p}\left(\omega^{\tilde{s} / \tilde{p}, \omega)}\right.}^{1 / r} \\
& \leq C\left\||f|^{r}\right\|_{L^{\tilde{p}, k}(\omega)}^{1 / r}=C\|f\|_{L^{p, k}(\omega)} .
\end{aligned}
$$

\section{Proof of main results}

First, we will give the proof of Theorem 2.1.

Proof of Theorem 2.1 Since $r_{\omega}>\frac{(n(1-\alpha)+2 \beta)(p-1)}{2 \beta p-n(1-\alpha)-2 \beta}$, there is $r$ such that $r>\frac{(n(1-\alpha)+2 \beta)(p-1)}{2 \beta p-n(1-\alpha)-2 \beta}$ and $\omega \in R H_{r}$. Then $p>\frac{(n(1-\alpha)+2 \beta)(r-1)}{2 \beta r-n(1-\alpha)-2 \beta}$. There exists $s$ such that $p>s>\frac{(n(1-\alpha)+2 \beta)(r-1)}{2 \beta r-n(1-\alpha)-2 \beta}>\frac{n(1-\alpha)+2 \beta}{2 \beta}$. The fact $s>\frac{(n(1-\alpha)+2 \beta)(r-1)}{2 \beta r-n(1-\alpha)-2 \beta}$ implies that $r>\frac{(n(1-\alpha)+2 \beta)(s-1)}{2 \beta s-n(1-\alpha)-2 \beta}$. By Lemma 3.3 and Lemma 3.12, we have

$$
\begin{aligned}
\| & {[b, T](f) \|_{L^{q, k q / p}\left(\omega^{1-q, \omega)}\right.} } \\
\leq & \left\|M_{t}([b, T] f)\right\|_{L^{q, k q / p}\left(\omega^{1-q, \omega)}\right.} \\
\leq & C\left\|M_{t}^{\sharp}([b, T] f)\right\|_{L^{q, k q / p}\left(\omega^{1-q, \omega)}\right.} \\
\leq & C\|b\|_{\operatorname{Lip}_{\beta_{0}}(\omega)}\left(\left\|\omega(\cdot) M_{\beta_{0}, s, \omega}(T f)\right\|_{L^{q, k q / p}\left(\omega^{1-q, \omega)}\right.}\right. \\
& \left.+\left\|\omega(\cdot) M_{\beta_{0}, s, \omega}(f)\right\|_{L^{q, k q / p}\left(\omega^{1-q, \omega)}\right.}\right) \\
= & C\|b\|_{\operatorname{Lip}_{\beta_{0}}(\omega)}\left(\left\|M_{\beta_{0}, s, \omega}(T f)\right\|_{L^{q, k q / p}(\omega)}+\left\|M_{\beta_{0}, s, \omega}(f)\right\|_{L^{q, k q / p}(\omega)}\right) .
\end{aligned}
$$

Therefore, applying Lemma 3.9 and Lemma 3.5, we obtain

$$
\begin{aligned}
\|[b, T](f)\|_{L^{q, k q / p}\left(\omega^{1-q, \omega)}\right.} & \leq C\|b\|_{\operatorname{Lip}_{\beta_{0}}(\omega)}\left(\|T f\|_{L^{p, k}(\omega)}+\|f\|_{L^{p, k}(\omega)}\right) \\
& \leq C\|b\|_{\operatorname{Lip}_{\beta_{0}}(\omega)}\|f\|_{L^{p, k}(\omega)} .
\end{aligned}
$$

This completes the proof of Theorem 2.1. 
Then we will give the proof of Theorem 2.2.

Proof of Theorem 2.2 It follows from $r_{\omega}>\frac{p-1}{p-q^{\prime}} q^{\prime}$ that $p>\frac{r_{\omega}-1}{r_{\omega}-q^{\prime}} q^{\prime}$. There exists $r$ such that $p>r>\frac{r_{\omega}-1}{r_{\omega}-q^{\prime}} q^{\prime}$. By Lemma 3.3 and Lemma 3.13, we have

$$
\begin{aligned}
&\|[b, T] f\|_{L^{s, k s / p}\left(\omega^{1-s, \omega)}\right.} \\
& \leq\left\|M_{\delta}([b, T] f)\right\|_{L^{s, k s / p}\left(\omega^{1-s, \omega)}\right.} \\
& \leq C\left\|M_{\delta}^{\sharp}([b, T] f)\right\|_{L^{s, k s / p}\left(\omega^{1-s, \omega)}\right.} \\
& \leq C\|b\|_{\operatorname{Lip}_{\beta_{0}}(\omega)}\left(\left\|\omega(\cdot) M_{\beta_{0}, r, \omega}(T f)\right\|_{L^{s, k s / p}\left(\omega^{1-s, \omega)}\right.}\right. \\
&+\left\|\omega(\cdot) M_{\beta_{0}, r, \omega}(f)\right\|_{L^{s, k s / p}\left(\omega^{1-s, \omega)}\right.} \\
&\left.+\left\|\omega(\cdot)^{1+\beta_{0} / n} M_{\beta_{0}, r}(f)\right\|_{L^{s, k s} /\left(\omega^{1-s, \omega)}\right.}\right) \\
&= C\|b\|_{\operatorname{Lip}_{\beta_{0}}(\omega)}\left(\left\|M_{\beta_{0}, r, \omega}(T f)\right\|_{L^{s, k s / p}(\omega)}\right. \\
&\left.+\left\|M_{\beta_{0}, r, \omega}(f)\right\|_{L^{s, k s / p}(\omega)}+\left\|M_{\beta_{0}, r}(f)\right\|_{L^{s, k s} / p\left(\omega^{s / p}, \omega\right)}\right) .
\end{aligned}
$$

Therefore, applying Lemma 3.9, Lemma 3.14 and Lemma 3.6, we obtain

$$
\begin{aligned}
\|[b, T] f\|_{L^{s, k s / p}\left(\omega^{1-s, \omega)}\right.} & \leq C\|b\|_{\operatorname{Lip}_{\beta_{0}}(\omega)}\left(\|T f\|_{L^{p, k}(\omega)}+\|f\|_{L^{p, k}(\omega)}\right) \\
& \leq C\|b\|_{\operatorname{Lip}_{\beta_{0}}(\omega)}\|f\|_{L^{p, k}(\omega)} .
\end{aligned}
$$

This completes the proof of Theorem 2.2.

Finally let us give the proof of the corollary.

Proof of Corollary 2.1 Choose $q$ such that $\max \left\{\frac{p r_{\omega}}{(p-1)\left(r_{\omega}-1\right)}, 2, p^{\prime}\right\}<q<\infty$, then $1<q^{\prime}<2$, $q^{\prime}<p$ and $q^{\prime}<\frac{p r_{\omega}}{p+r_{\omega}-1}$. It follows from $q^{\prime}<\frac{p r_{\omega}}{p+r_{\omega}-1}$ that $r_{\omega}>\frac{(p-1) q^{\prime}}{p-q^{\prime}}$.

Since $T$ is a generalized Calderón-Zygmund operator defined as in Definition 1.2 with $C_{j}=2^{-j \delta}(j \in \mathbf{N})$ and this pair of numbers $\left(q, q^{\prime}\right)$, then by Theorem 2.2 we have that $[b, T]$ is bounded from $L^{p, k}(\omega)$ to $L^{s, k s / p}\left(\omega^{1-s}, \omega\right)$.

\section{Competing interests}

The authors declare that they have no competing interests.

\section{Authors' contributions}

The authors contributed equally and significantly in writing this paper. All authors read and approved the final manuscript.

\section{Acknowledgements}

This work was supported by the National Natural Science Foundation of China (11171345), Beijing Higher Education Young Elite Teacher Project (YETP0946) and the Fundamental Research Funds for the Central Universities (2009QS16).

Received: 18 August 2015 Accepted: 8 October 2015 Published online: 22 October 2015

\section{References}

1. Fefferman, C, Stein, EM: HP Spaces of several variables. Acta Math. 129, 137-193 (1972)

2. Coifman, R: A real variable characterization of $H^{p}$. Stud. Math. 51, 269-274 (1974)

3. Alvarez, J, Milman, M: HP Continuity properties of Calderón-Zygmund type operators. J. Math. Anal. Appl. 118, 63-79 (1986)

4. Alvarez, J, Milman, M: Vector valued inequalities for strongly Calderón-Zygmund operators. Rev. Mat. Iberoam. 2 , 405-426 (1986) 
5. Lin, Y, Lu, SZ: Toeplitz operators related to strongly singular Calderón-Zygmund operators. Sci. China Ser. A 49, 1048-1064 (2006)

6. Lin, Y, Lu, SZ: Boundedness of commutators on Hardy-type spaces. Integral Equ. Oper. Theory 57, 381-396 (2007)

7. Lin, Y, Lu, SZ: Strongly singular Calderón-Zygmund operator and their commutators. Jordan J. Math. Stat. 1, 31-49 (2008)

8. Chang, DC, Li, JF, Xiao, J: Weighted scale estimates for Calderón-Zygmund type operators. Commun. Contemp. Math. $445,61-70(2007)$

9. Lin, Y: Sharp maximal function estimates for Calderón-Zygmund type operators and commutators. Acta Math. Sci. Ser. A Chin. Ed. 31, 206-215 (2011)

10. Morrey, CB: On the solutions of quasi-linear elliptic partial differential equations. Trans. Am. Math. Soc. 43, 126-166 (1938)

11. Campanato, S: Propietà di inclusione per spazi di Morrey. Ric. Mat. 12, 67-86 (1963)

12. Peetre, J: On the theory of $L_{p \lambda}$ spaces. J. Funct. Anal. 4,71-87 (1969)

13. Chiarenza, F, Frasca, M: Morrey spaces and Hardy-Littlewood maximal function. Rend. Mat. Appl. 7, 273-279 (1987)

14. Fu, ZW, Lu, SZ: Weighted Hardy operators and commutators on Morrey spaces. Front. Math. China 5, 531-539 (2010)

15. Lin, Y: Strongly singular Calderón-Zygmund operator and commutator on Morrey type spaces. Acta Math. Sin. 23, 2097-2110 (2007)

16. Komori, Y, Shirai, S: Weighted Morrey spaces and a integral operator. Math. Nachr. 282, $219-231$ (2009)

17. Wang, H: Some estimates for commutators of Calderón-Zygmund operators on weighted Morrey spaces. Sci. Sin., Math. 42, 31-45 (2012)

18. Shi, SG, Fu, ZW, Zhao, FY: Estimates for operators on weighted Morrey spaces and their applications to nondivergence elliptic equations. J. Inequal. Appl. 2013, 390 (2013)

19. Lin, Y, Sun, GF: Strongly singular Calderón-Zygmund operators and commutators on weighted Morrey spaces. J. Inequal. Appl. 2014, 519 (2014)

20. Lin, Y, Sun, GF: Generalized Calderón-Zygmund operators and commutators on weighted Morrey spaces. Panam. Math. J. 25, 53-65 (2015)

21. Muckenhoupt, B: Weighted norm inequalities for the Hardy maximal function. Trans. Am. Math. Soc. 165, 207-226 (1972)

22. Garcia-Cuerva, J, Rubio de Francia, JL: Weighted Norm Inequalities and Related Topics. North-Holland, Amsterdam (1985)

23. Journé, JL: Calderón-Zygmund Operators, Pseudo-Differential Operators and the Cauchy Integral of Calderón. Lecture Notes in Math., vol. 994, pp. 1-127 (1983)

24. Garcia-Cuerva, J: Weighted $H^{p}$ spaces. Diss. Math. 162, 1-63 (1979)

25. Paluszyński, M: Characterization of the Besov spaces via the commutator operator of Coifman, Rochberg and Weiss. Indiana Univ. Math. J. 44, 1-17 (1995)

\section{Submit your manuscript to a SpringerOpen ${ }^{\circ}$ journal and benefit from:}

- Convenient online submission

- Rigorous peer review

- Immediate publication on acceptance

- Open access: articles freely available online

- High visibility within the field

- Retaining the copyright to your article 